\title{
A 3-D Self-Reconfigurable Structure
}

\author{
Satoshi Murata*, Haruhisa Kurokawa, Eiichi Yoshida, Kohji Tomita and Shigeru Kokaji \\ Mechanical Engineering Laboratory, AIST, MITI \\ 1-2 Namiki, Tsukuba, 305 Japan, Email: murata@mel.go.jp
}

\begin{abstract}
A three-dimensional, self-reconfigurable structure is proposed. The structure is a fully distributed system composed of many identical 3-D units. Each unit has functions of changing local connection, information processing, and communication among neighborhood units. Groups of units cooperate to change their connection so that the shape of the whole solid structure transforms into arbitrary shape. Also, the structure can repair itself by rejecting faulty units, replacing them with spare units. This kind of self-maintainability is essential to structure's longevity in hazardous or remote environments such as space or deep sea, where human operators cannot approach. We have designed and built a prototype unit to examine the feasibility of the 3-D self-reconfigurable concept. The design of the unit, method of reconfiguration, hardware implementation, and results of preliminary experiments are shown. In the last part of this paper, distributed software for self-reconfiguration is discussed.
\end{abstract}

\section{Introduction}

The purpose of this paper is to demonstrate feasibility of 3-D self-reconfigurable structure. We have been studying homogeneous distributed mechanical systems, in which all elements of the system are identical. The 3-D selfreconfigurable structure is one realization of our concept.

The concept of a homogeneous system, which is similar to cell structure of biological organisms, has fundamental applications and advantages in the design of large, complex artifacts. In the quest for such a system, the following advantages are considered: 1) Universal design: if one can design a universal unit, theoretically at least, one can construct virtually anything by combination of the units. 2) High productivity: homogeneous unit is suitable for mass production. 3) Low maintenance cost: The system can easily repair itself by replacing units in a cost effective way. 4) Self-assembly and self-repair: if the homogeneous unit has the ability to change local inter-unit connection, then the group of such units can transform into any desired shape without external assistance. Moreover, the system can repair itself by ejecting faulty units and reassembling the structure.

This kind of self-reconfigurable system is very versatile, because one can almost freely design various configurations through combinations of the units. One can not only build static structures, but also dynamic mechanical systems (e.g., locomotion systems, manipulators etc.). Such next-generation mechanical systems that incorporate selfmaintainability have fundamental applications in hazardous or remote environments, including space, deep sea and radioactive environments. For instance, a large space structure can be built by this method. It provides not only the primary structure of the space station, but large variety of shapes and functions are available, such as antennas, solar panels, manipulators and so on. The structure changes its shape and function according to its mission, and even if some part contains a damage or malfunction, it is still possible to maintain the total function by the automatic reconfiguration.

In this paper, we propose a $3-\mathrm{D}$ reconfigurable mechanical system, consisting of homogeneous units. To the authors' knowledge, this is the first feasible 3-D system with self-reconfigurability. The basic design of the homogeneous 3-D unit, method of self-reconfiguration, and detail of the mechanism will be described. Experimental results from a prototype unit are presented. Also, we provide some preliminary result of distributed software for selfreconfiguration.

\section{Related Research}

There have been numerous studies of the selfreconfigurable system. However, a systematic design method is yet to be established. The conventional studies on self-reconfigurable systems can be classified into three general categories.

The first category consists of pure mathematical models, usually described in a cellular-automata form. Spatiotemporal pattern development in the cell-space is the main subject in this field. One can trace back to Von Neumann's self-reproducing automata [1] as the origin of cellular automata models. There are many variations of his model [2]. Nevertheless, it is difficult to construct these models as real hardware. Recently, Beni developed a more feasible cell model, and discussed the problem of shape 
formation [3].

Unit-structured system with fixed configuration comprises the second category. Such systems are made of many homogeneous/heterogeneous mechanical units, but these units must be manually combined. Various machines, such as a legged walking machine and a manipulator system, have been constructed by the method $[4,5]$.

The third category covers unit-structured systems with dynamic reconfiguration ability --- the main topic of this paper. Thus far, several reconfigurable systems have been developed. However, all are one- or two-dimensional systems $[6,7,8]$. Chirikjian et al. developed several versions of a modular robotic system, made of homogeneous 2-D units $[9,10]$. Each of their unit is a hexagonal link with 6 servo motors. These units can dynamically change connection with neighboring units and climb over an adjacent unit. Our 2-D system is similar system, but has a much simpler mechanism [11,12]. Each unit, called a "fractum," has three electromagnets for actuation, an onboard micro-processor, and optical communication devices for local information exchange. For this hardware, we have developed several algorithms for 2-D self-assembly and self-repair, and these are examined by hardware experiments [13].

To the authors' knowledge, no three-dimensional reconfigurable units exist so far. Because, there are some basic difficulties to extend these two-dimensional concept to the three-dimensional space.

One of main difficulties is the gravity field. All of the above two-dimensional unit can generate very small torque, but it is enough to carry the unit against friction between the unit and floor. However, in the three-dimensional case, the unit must lift up another unit with the same weight in the field of gravity, and this requires some well-designed mechanism with high power/weight ratio.

Another difficulty comes directly from dimensions and is more essential. In the two-dimensional case, the unit is actually built in three dimensional space. Thus, the horizontal area which a unit can occupy is determined from planer geometrical symmetry of the structure, but the vertical space is remained free for a designer. In the threedimensional case, there are no such residual dimension, which makes the design very difficult.

\section{Unit Design and Method of Reconfiguration}

In 3-D system design, the homogeneity of the system is a very stringent and difficult requirement for a designer. Each unit must have spatial symmetry. In other words, the shape and function of the unit must be the same for each symmetry axis. To achieve a 3-D system, one needs at least three symmetry axes, and the unit must posses some degree of mobility around each axis.

As a starting point of design, we selected several space filling polyhedra (Fig.1). The rhombic dodecahedron (a) and the truncated octahedron (b) can be thought as a type of extension of a 2-D hexagon to 3-D space. We chose them because we have successful 2-D unit design based on a hexagon [11]. However, a feasible solution has not been found. Another candidate is the regular hexahedron (c), which is one of the most simple space filling polyhedra. Each of three symmetry axes of this polyhedron is orthogonal to the others, which is a great advantage in the design of mechanisms.

Consequently, the regular hexagon was selected as the base geometry, thereupon, hardware of the 3-D unit is founded. The unit consists of a cube at the center and six connecting arms attached to six sides of the cube (Fig.2). The unit can rotate each connecting arm independently, and each connecting arm has a connecting mechanism at its end. By using the connecting mechanism, the unit can either connect or disconnect its arm to that of another units. Arbitrary cubic structures (like a "jungle gym") are possible by this inter-unit connection.

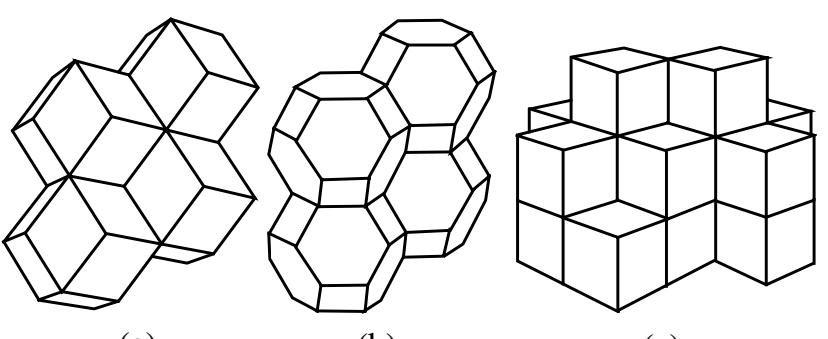

(a)

(b)

(c)

Fig.1 Space-filling polyhedra



Fig.2 Basic structure of 3-D unit 
The structure is reconfigurable by means of the rotating arms and the connecting mechanism. There are two basic methods of reconfiguration: one is movement on a plane, the other is transfer to orthogonal plane. In both method, the unit always makes motion accompanied with another unit. Basically, one can build arbitrary 3-D structures by appropriate combination of these basic movements.

Figure 3 shows how two units move on a plane made of many other units. One unit plays the role of a pivot. The other unit releases its connection to the plane and the pivot unit carries the partner to another position. After the partner unit reconnects to the plane, it then becomes a pivot in turn. By repeating this process, a pair of units can move to an arbitrary position on the plane.

Figure 4 illustrates the second method, how to transfer to an orthogonal plane. Before applying this method, a pair of units must stand on the edge of the plane A. Then a unit under the pair plays the role of a pivot and carries one unit of the pair to the orthogonal plane B. By repeating this motion for the rest of the pair, we can transfer the pair onto the plane B.

Pair-wise movement is a key idea of the method. Of

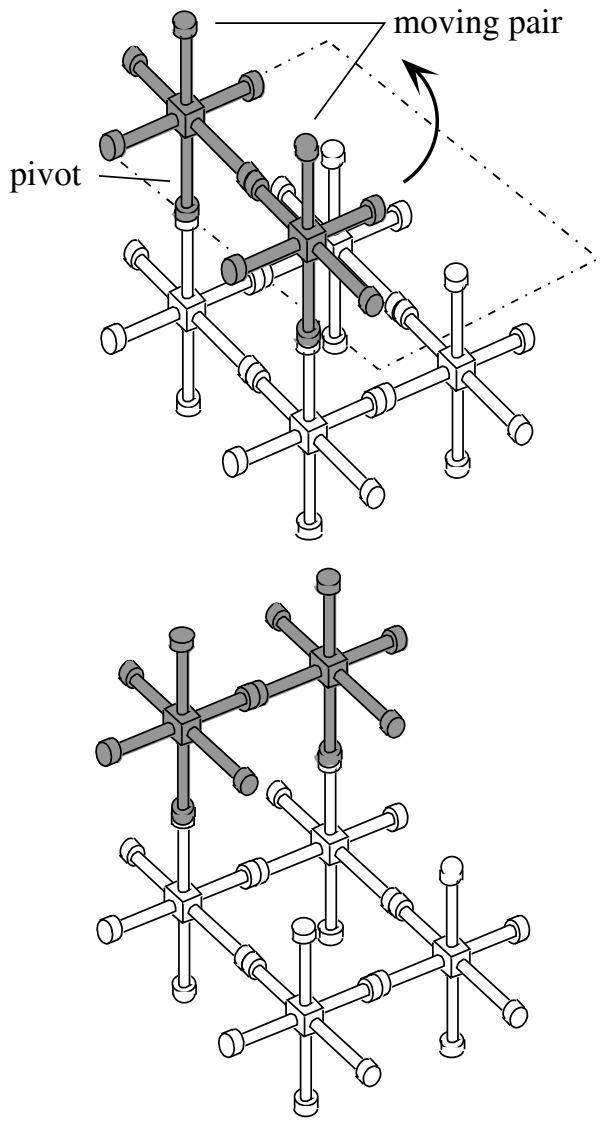

Fig.3 Pair-wise movment on a plane significance, this method has advantages when compared to another more straightforward approach. Figure 5 shows a type of unit with sliding mechanism. Each side of the unit is equipped with some sliding mechanism which can move the unit in two directions. Thus the unit needs two degrees of freedom for each side in this case. Compared with this, our method based on pair-wise motion requires only one rotational degree of freedom.

By the 3-D unit and pair-wise motion, we can build an almost arbitrary structure, but there are some exceptions. One is called "a checker board limitation." Consider a 3-D checker board. If a unit is put on a black node at the initial time, it never moves to white nodes. Thus, the black/white distribution of the initial state is always conserved. This kind of limitation disappears, if we regard a pair of the unit as the primitive element of the structure. Thus, if the number of the unit involved in the structure is even, then the checker

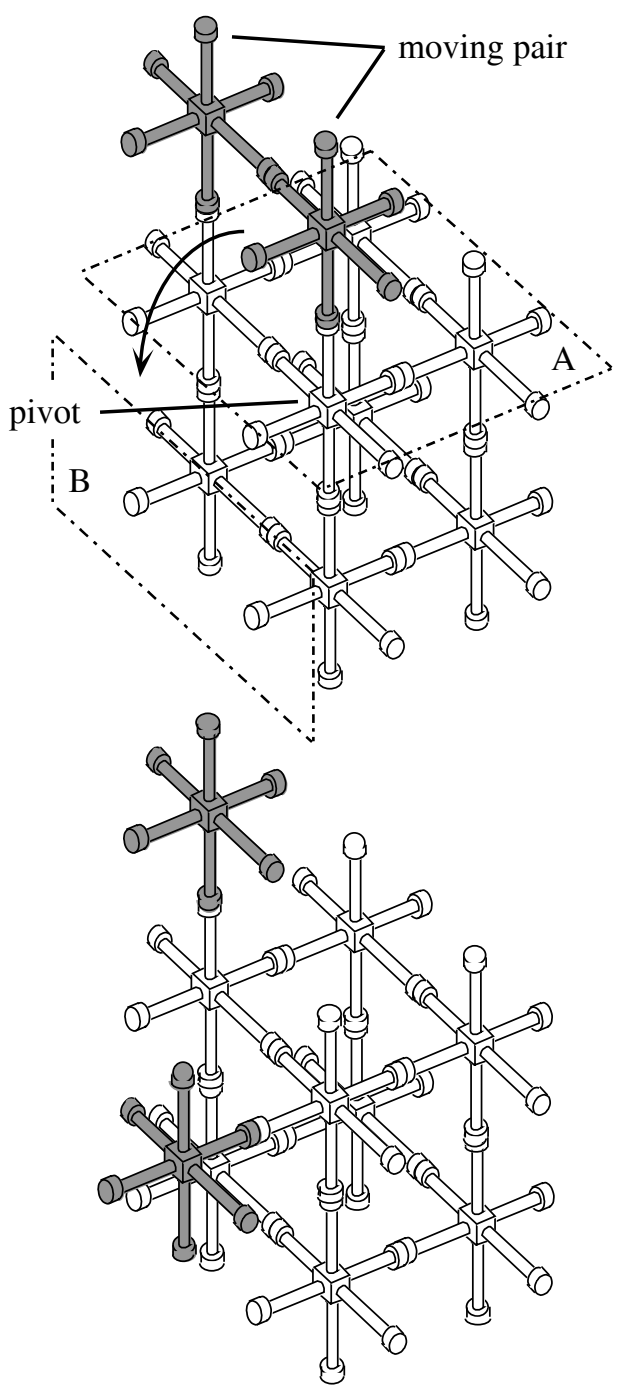

Fig.4 Transfer to an orthogonal plane 


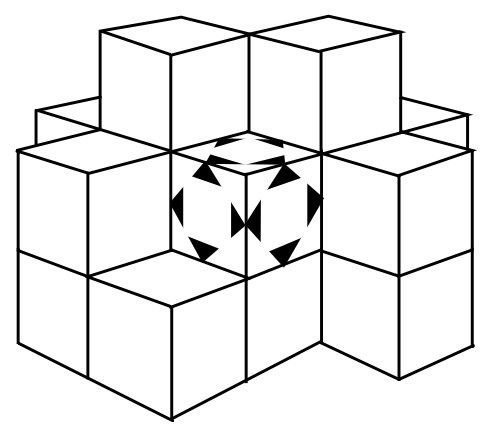

Fig.5 A unit performs translational motion

board limitation never arises. Even if the number of the unit is odd, this limitation is not essential in most cases of real application, where sufficient number of the unit exist.

We must recognize another limitation; there are some difficult configurations to be built by the pair-wise motion. Typical of such configurations is a linear string of the unit. They can rotate coaxially, but cannot change the configuration. However, if we put a pair of units on the line, then they can start assembly (Fig. 6). Thus, most of the configuration can be transferred into other configurations, and this comprises a set of reachable configurations. The classification of reachable configuration set from one specific configuration is one of interesting

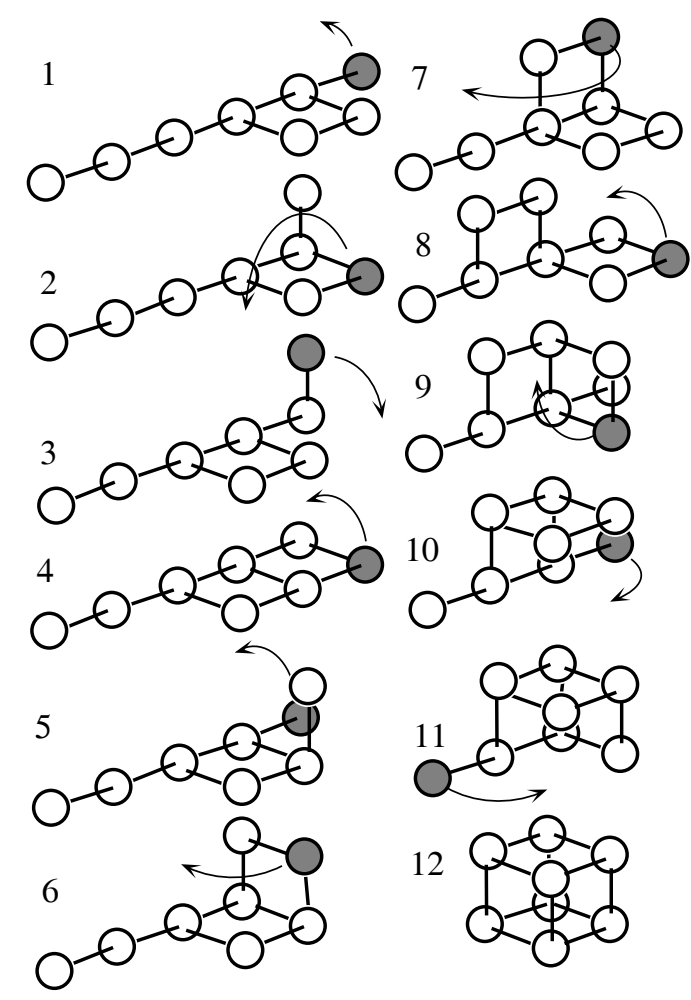

Fig.6 Sequence of reconfiguration from a line to a cube. A shaded unit moves in the next step. issues in reconfigurable system [10]. The classification in the three-dimensional case (especially utilizing our unit structure) is under investigation.

\section{Hardware Implementation}

Hardware was developed according to the basic design criteria discussed in the previous section. From a technical standpoint, there are several additional requirements in hardware design.

Compact and simple implementation: The unit needs 12 degrees of freedom, six for rotation of arms, and six for connecting/disconnecting of arms. Each degree of freedom must be controlled independently. We need to design a simple and compact form.

Complemental connecting mechanism: The connecting mechanism requires a kind of "key and key hole" relationship. But we cannot use a pair of male and female connectors because of the homogeneity and symmetry restrictions. Therefore, the shape of connecting mechanism must be self-complemental; a concave surface of the connecting mechanism must correspond to a convex surface of itself.

High power-to-weight ratio: To achieve the reconfiguration on earth, each unit must generate enough torque to be able to lift another unit.

Geometric precision: Relative positioning among the units is very important in the basic movement. Thus, the connection mechanism between the units must be sufficiently stiff to maintain a precise geometric relationship. Furthermore, the mechanism must absorb deviation from the normal position.

Energy/information transmission: The power supply line and communication channel must be connected through the connecting arm.

After having considered these additional requirements, we designed the following mechanism (Fig.7). An actuator, torque transmission system and a micro-processor are contained by the central cubic body. Only one motor used in each unit. In addition, the motor can change the power transmission route to select one of the 12 degrees of freedom by electrical clutches. Connection between the arms is accomplished by a special "handshake" mechanism that provides a very stiff link between the units. High-torque rotation mechanism of the arm is provided by a worm gear/ wheel.

\section{Actuator and torque transmission system}

Actuator and torque transmission system is illustrated in Fig. 8. We adopted a combination of a DC motor and a harmonic drive as a power source. The output of the 


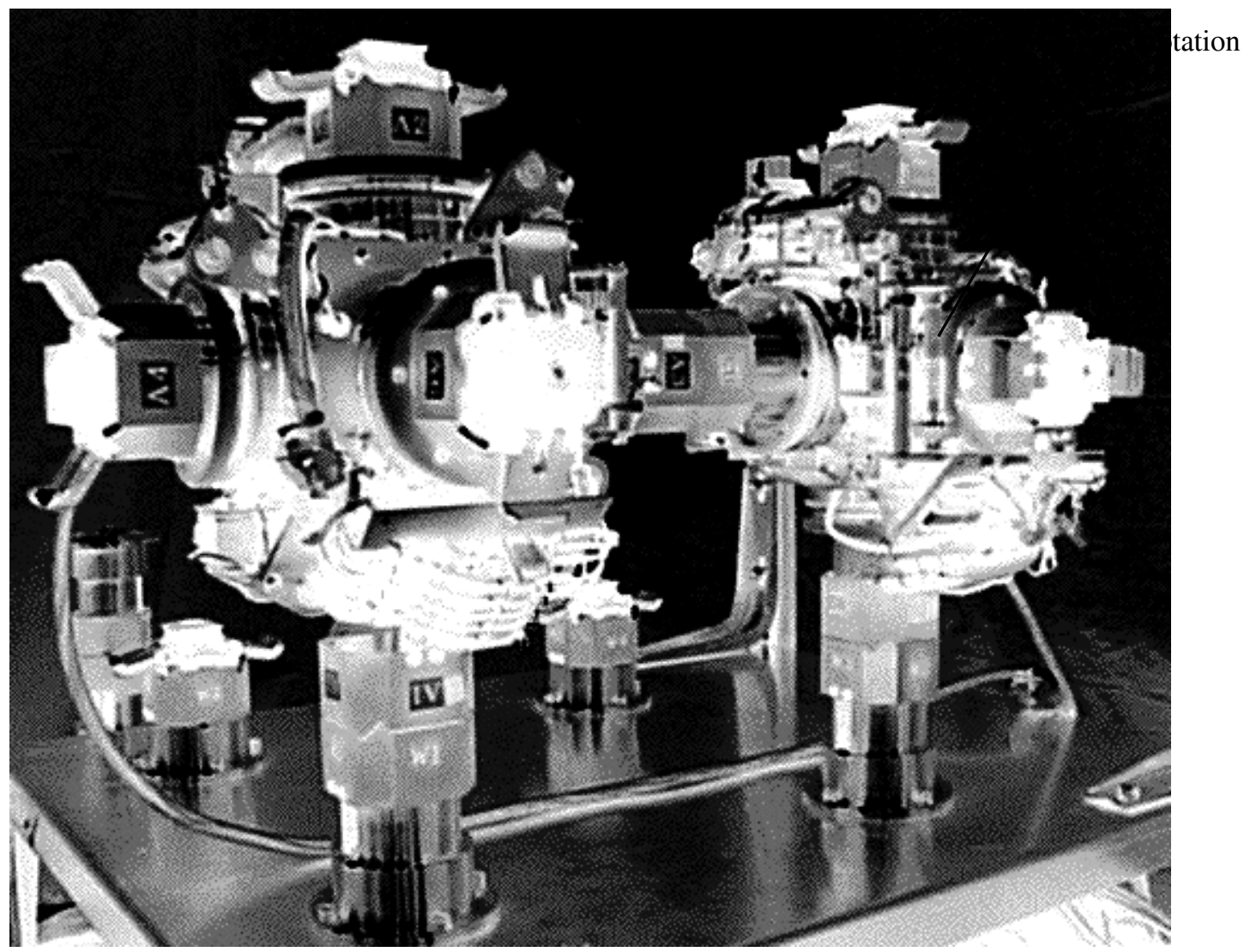

Fig.7 3-D Unit and experimental apparatus

harmonic drive is delivered to four main transmission shafts (located in the corners of the cube) using a timing belt. Each side of the cube needs one axis for the handshake mechanism and another axis for the arm rotation (omitted in Fig.8). The total of 12 axes are connected to the main transmission

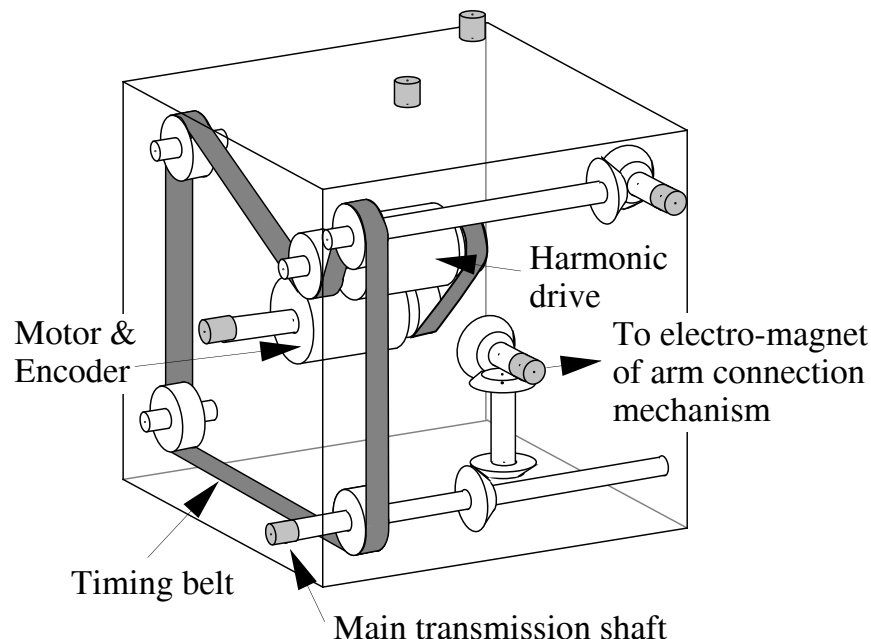

Fig.8 Torque transmission system*

*Worm gear system for arm rotation is connected to one of main transmission shaft or harmonic reduction gear, but omitted in this figure. See also Fig.7. shafts or connected directly to the output axis of the harmonic drive. The DC motor is equipped with a rotational encoder for position control.

\section{Arm rotation mechanism}

We use the worm gear and wheel combination as the rotation mechanism of the arm. This mechanism can generate high torque and hold the rotation angle without inputting energy to the motor. For control, each worm gear is connected to the main transmission shaft through a switching mechanism driven by a solenoid. The rotation angle of the connection arm is measured by the rotary encoder attached to the motor and an electric contact sensor for initial positioning.

\section{Arm connection mechanism}

Figure 9 shows the section of the handshake mechanism. The mechanism consists of a connection hand, a connection head, and a connection cuff. The connection cuff slides back and forth along a screw shaft in the arm which is connected to the main transmission shaft through an electromagnetic clutch. When the connection cuff slides forward to the end of travel, it pushes up the connection hand to close, and the hand grasps another connection arm's head. Connection completes when the connection cuff slides 


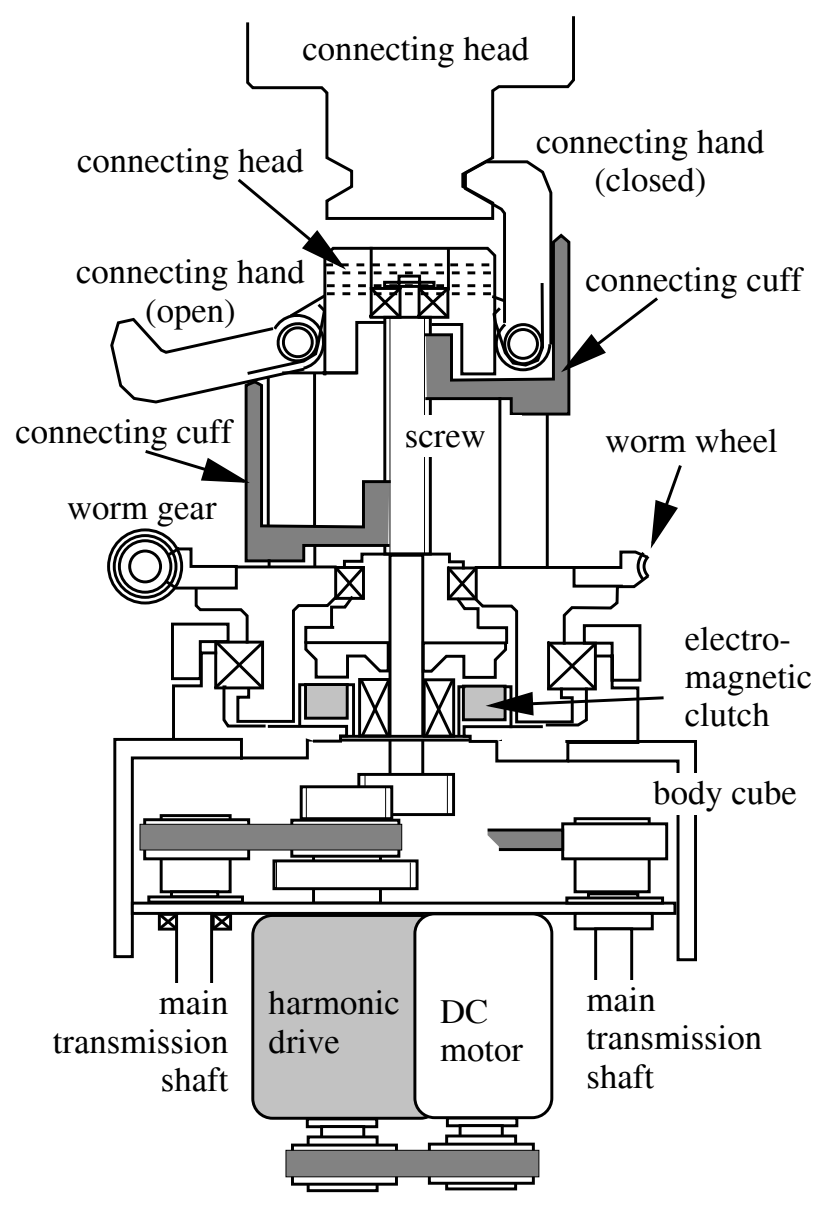

Fig.9 Connecting arm mechanism

to the utmost position pressing another arm's cuff (Fig.10). This connecting mechanism is very stiff for bending stress. It can also absorb small positional deviation by the grasping motion of the connection hand. Contact points for energy/ information transmission can be embedded in the connection hand and the connection head.

\section{Experiments}

Dimensions of the prototype unit are summarized as follows. The span (i.e., the distance between the adjacent unit center) of the unit is $26.5 \mathrm{~cm}$ and it weighs approximately $7 \mathrm{Kg}$. It includes one DC motor (7 W), a harmonic drive (reduction ratio: 1/100), six solenoids for arm rotation control and six electromagnetic clutches for connection control. A rotary encoder is attached to the motor to measure the position of rotation arm and connection hand. It also has six small contact sensors at the root of each connection arm to set it to the origin. The onboard microprocessor and the electric connectors for energy/information transmission is not installed in the current model.

The experimental apparatus consists of two fully-
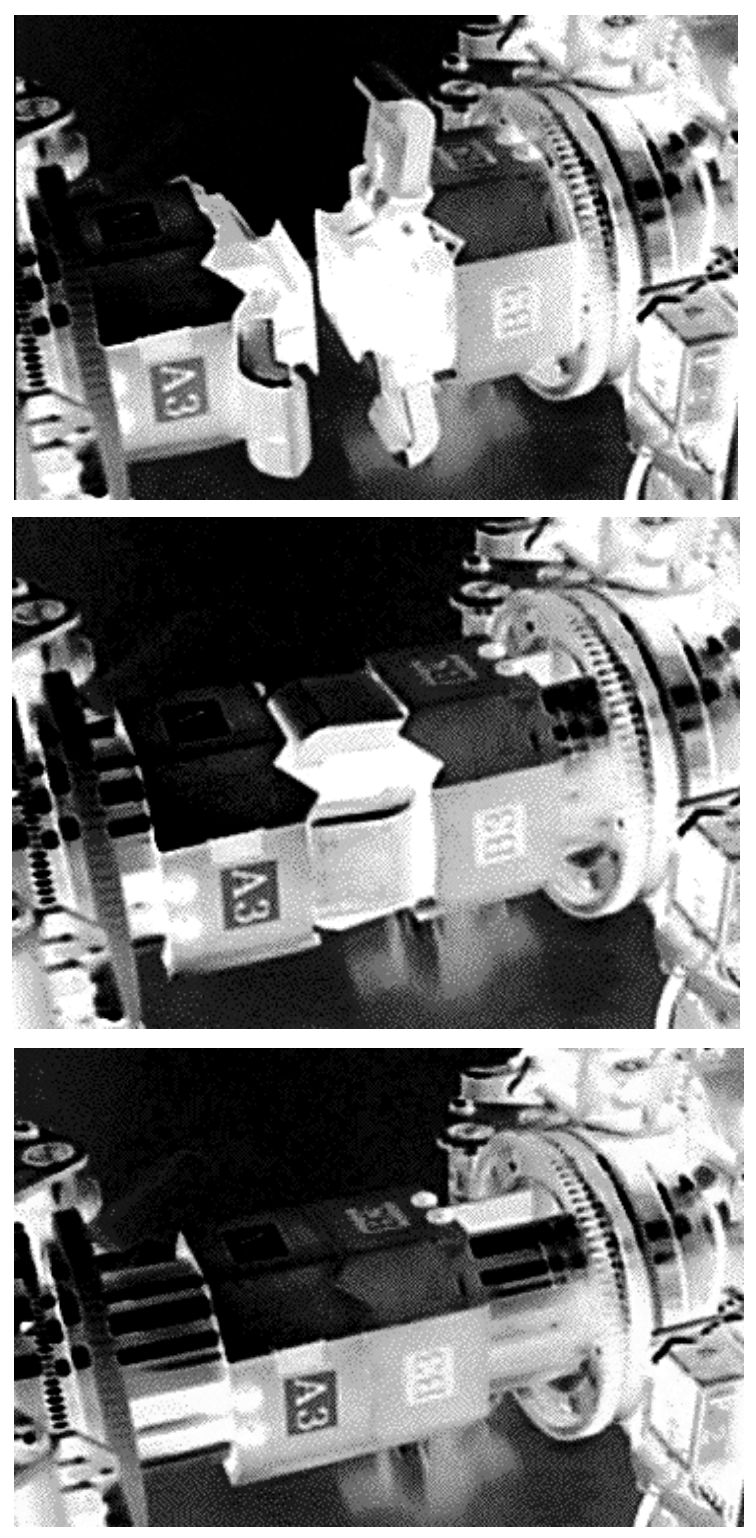

Fig.10 Sequence of arm connection

equipped prototype units and a reference plane with four connection hands.

We have verified basic functions of the unit using the apparatus. To evaluate pair-wise rotational movement in various postures, we conducted lifting tests. The unit satisfactorily demonstrated its ability to raise up another unit. The reference plane in Fig.7 was vertically set for this experiment. In this condition, the speed of rotation was about $6 \mathrm{deg} / \mathrm{sec}$, and it was not significantly affected by the posture. The connection mechanism has also been tested in various postures. Gravity causes positional deviation between ends of the connection arms. In the worst case, the deviation approaches $10 \mathrm{~mm}$, but the mechanism could absorb the deviation and establish firm connection. 


\section{Distributed Reconfiguration Algorithm}

We are currently investigating a distributed reconfiguration algorithm for 3-D units in parallel with the hardware development. The algorithm is based on a simple expression of connection description and a stochastic relaxation method to cope with many degrees of freedom of 3-D system. It is implemented in such a way that the homogeneity is preserved also in the software aspect.

\section{Target Shape Description}

To describe target shape, we first introduce a simple classification of "connection types" of units as shown in Fig.11. As a result, we have nine connection types by neglecting rotational transformations.

Next, we explain how to express target shapes. Suppose a cube composed of eight units (Fig.12 (a)). Each unit has C31 type and is connected to three C31 types, so this situation is written as a "type-list" C31[C31, C31, C31]. We describe the target shape as a collection of these typelists, so the 8-unit cube is straightforwardly given as

$$
\text { C31[C31, C31, C31] . }
$$

The 12-unit box shape in Fig.12(b) is given by two type-lists:

$$
\begin{aligned}
& \text { C31[C41, C31, C31], } \\
& \text { C41[C41, C41, C31, C31] . }
\end{aligned}
$$

In this way, the target shapes can be expressed using this connection types in a local manner. The homogeneity is preserved by distributing these descriptions to each unit.

\section{Self-reconfiguration Algorithm}

The algorithm is characterized by a stochastic

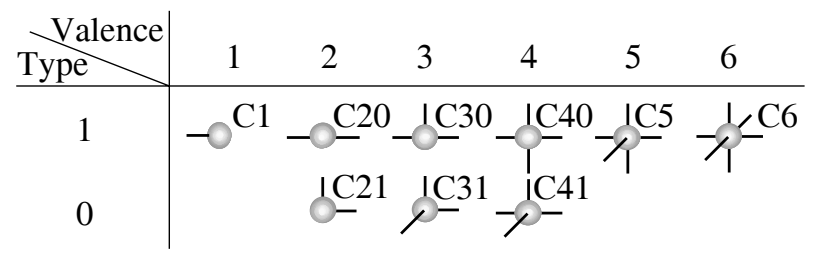

Fig.11 Connection types of 3-D units

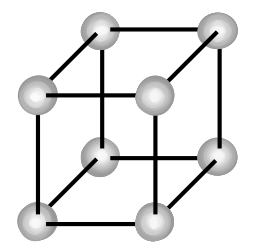

C31 [C31, C31, C31]

(a)

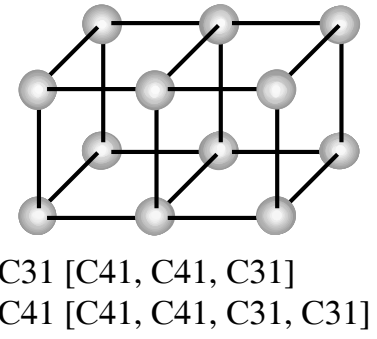

(b)
Fig.12 Description of target shape relaxation process, which allows the unit system to converge to a given target shape by searching for a proper unit motion over many degrees of freedom.

An alternative solution is a heuristic algorithm like if [configuration]-then [motion] rules. However, we are forced to provide ad hoc heuristics for every different target shapes. Since we intend to make the algorithm simple and general, we adopted the above connection type notation and a stochastic process.

In reconfiguration process, we allow only units with limited connection types to move, which are referred to "movable types" to maintain the connectivity of the whole unit system. Types C1, C21, and C31 are selected as movable types here. "Reachable positions" denotes the lattice points a unit can reach by pair-wise motion in Figs. 3 and 4 . The algorithm consists of the following three procedures: Each unit

(i) tests whether it is movable and, if so, calculates all the reachable positions through local communication.

(ii) calculates the "difference" $D$ between the current and the goal configuration for all reachable positions, and

(iii) moves with a greater probability in the direction of smaller potential calculated from the difference $D$.

We call this series of procedures a "control step". This control step is assumed to be synchronous in all the units.

In the procedure (ii) in the control step, for each reachable position $i$ (including "no motion" as $i=0$ ), each unit each unit computes the difference $D_{i}$ from target shape. It is calculated using an expansion of the method developed for the 2-D fracta system [11][14]. We leave the details to our related paper in preparation. In short, the value $D_{i}$ represents the difference between the state at reachable position $i$ and the closest type-list in the target description. The zero $D_{i}$ value means that one of the target type-list is completed at the reachable position $i$.

Next in (iii), the probability $P_{i}$ that a unit moves to the reachable position $i$ is computed based on Markov Random Field [14] using the difference $D_{i}$ as a potential function. Besides the convergence to the minimum potential, this method has another advantage of parallel implementation using local information. The MRF-based probability is given by:

$$
P_{i}=\frac{1}{Z} \exp \left(-\frac{D_{i}}{T}\right), \quad Z=\sum_{i} \exp \left(-\frac{D_{i}}{T}\right)
$$

where the parameter $T$ corresponds to artificial temperature in the simulated annealing method. By decreasing $T$ gradually, units tries possible reachable positions uniformly in earlier steps. As time elapses, the units come to make more concentrated motion to the closest position to complete 
goal structure.

The above relaxation computation allows the whole system to converge to the target shape even though there are many alternatives of movement.

\section{Simulation}

The effectiveness of the proposed algorithm is evaluated by computer simulations here. The target shape in Fig.12(b) is constructed from given initial configurations in simulations. The algorithm has been implemented in a distributed and parallel way.

In the following simulation, only one unit is allowed to move at each step for simplicity. Figure 13 shows a graphical view of simulation sequences of reconfiguration process, starting from the ladder shape. In this example, the system completed the self-assembly in 70 steps. This algorithm gives good performance for up to twenty units to construct such target shapes consisting of cubes as those in Fig.13. Construction of more complex structure will be addressed in future studies.

\section{Conclusion}

In this paper, a concept of 3-D self-reconfigurable structure has been proposed, and a method for selfreconfiguration has been developed. Corresponding hardware implementation has demonstrated. The basic
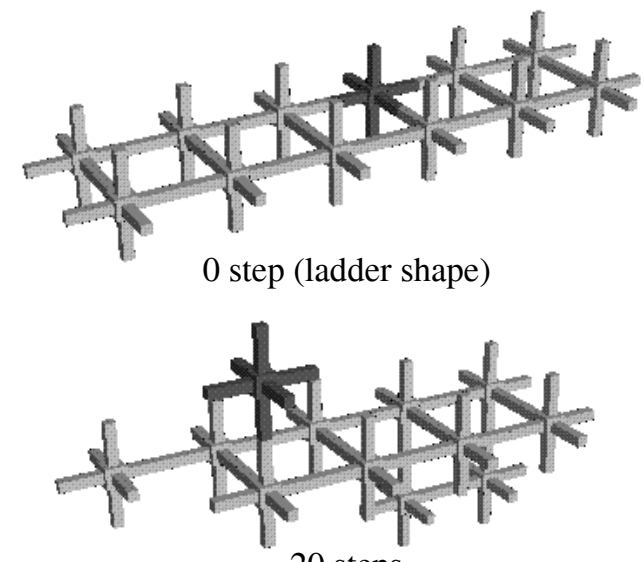

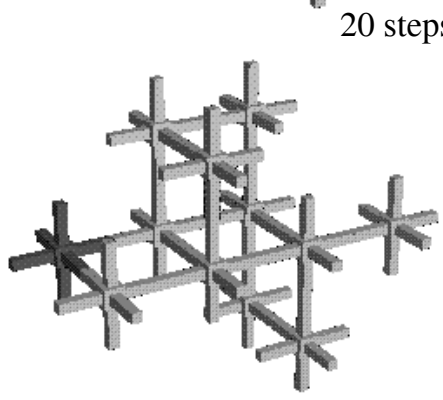

40 steps

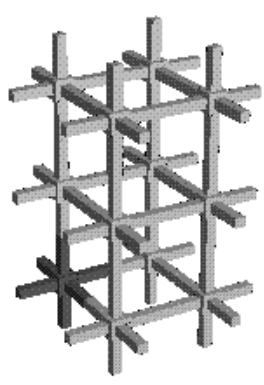

70 steps
Fig.13 Simulation of self-reconfiguration mechanical functions of the 3-D unit hardware was empirically studied. Along with the hardware development, software for self-assembly is also investigated. A simple algorithm of self-assembly on the basis of complete homogeneity and local communication is presented.

Self-reconfigurability means the system can maintain itself without outside help. Therefore, the technique is essentially important for autonomous mechanical systems used in hazardous or remote environments, such as space and deep sea. In particular, it may solve various problems in space, for instance, structural failures of solar panel expansion.

\section{References}

[1] J.von Neumann: Theory of Self-reproducing Automata, Univ. of Illinois Press (1966)

[2] C.G.Langton: Self-reproduction in Cellular Automata, Cellular Automata (1984)

[3] G.Beni: Research Perspectives in Swarm Intelligence, The Reconfiguration Problem, Proc. JSME System Life, $51 / 59$ (1997)

[4] M.Yim: New Locomotion Gaits, Proc. IEEE Intl. Conf. Robotics and Automation, 1508/1524 (1994)

[5] G.J.Hamlin, A.C.Sanderson: TETROBOT Modular Robotics: Prototype and Experiments, Proc. IEEE/RSJ Intelligent Robots and Systems, 390/395 (1996)

[6] L.S.Penrose: Self-reproducing, Sci. Amer., 200-6, 105/ 114 (1959)

[7] T.Fukuda et al.: Concept of Cellular Robotic System (CEBOT) and Basic Strategies for its Realization, Computers Electro. Eng., 18-1, 11/39 (1992)

[8] K.Hosokawa, I.Shimoyama, H.Miura: 2-D Micro-SelfAssembly using the Surface Tension of Water, Sensors and Actuators A, Vol. 57, 117/125 (1996)

[9] G.S.Chirikjian: Kinematics of Metamorphic Robotic System, Proc. IEEE Robotics and Automation, 449/455 (1994)

[10] G.S.Chirikjian, A.Pamecha, I.Ebert-Uphoff: Evaluating Efficiency of Self-Reconfiguration in a Class of Modular Robots, J.Robotic Systems, 13-5, 317/338 (1996)

[11] S.Murata, H.Kurokawa, S.Kokaji: Self-Assembling Machine, Proc. IEEE Robotics and Automation, 441/ 448 (1994)

[12] K.Tomita et al.: Reconfiguration Method for a Distributed Mechanical System, Distributed Autonomous Robotic System 2 (Ed. Asama et al.), 17/ 25, Springer-Verlag (1996)

[13] E.Yoshida et al.: Distributed Formation Control for a Modular Mechanical System, Proc. IEEE/RSJ Intelligent Robots and Systems, 1090/1097 (1997)

[14] S.Geman and D.Geman : Stochastic Relaxation, Gibbs Distributions, and the Baysian Restoration of Images, IEEE. Trans. Pattern Analysis and Machine Intelligence, 6-6, 721/741 (1984) 